DOI: $10.20396 /$ etd.v20i4.8649913

\title{
UTILIZAÇÃO DE NARRATIVAS DIGITAIS EM AMBIENTES DE APRENDIZAGEM BASEADA EM JOGOS DIGITAIS: UMA REVISÃO SISTEMÁTICA DA LITERATURA
}

\author{
THE USE OF DIGITAL STORYTELLING IN DIGITAL GAME-BASED LEARNING \\ ENVIRONMENTS: A SYSTEMATIC REVIEW OF THE LITERATURE
USO DE DIGITAL STORYTELLING ENTORNOS DE APRANDIZAJE BASADO EM JUEGOS DIGITALES: UNA REVISIÓN SISTEMÁTICA DE PUBLICACIONES

\author{
Ricardo Luiz Aoki ${ }^{1}$, Patricia Jantsch Fiuza ${ }^{2}$, Robson Rodrigues Lemos ${ }^{3}$
}

\begin{abstract}
RESUMO
A utilização de jogos digitais como suporte aos processos de ensino e aprendizagem tem sido um campo de pesquisas em amplo desenvolvimento. E o estudo das Narrativas Digitais é parte fundamental do entendimento de como os jogos digitais atuam nos processos cognitivos dos estudantes. Este artigo é resultado de uma Revisão Sistemática de Literatura que possibilitou uma meta-análise de 28 estudos que apresentam correlação da utilização da teoria de Narrativas Digitais com a Aprendizagem Baseada em Jogos Digitais. Conclui-se que estudos futuros respondam como a Narrativa Digital aplicada em jogos digitais educativos inferem na reflexão do sujeito e possam contribuir para que o mesmo obtenha o aprendizado de forma eficaz.
\end{abstract}

PALAVRAS-CHAVE: Narrativa Digital. Aprendizagem Baseada em Jogos Digitais. Jogos Digitais.

\begin{abstract}
The use of digital games to support the teaching and learning processes has been a field of research in widespread development. And the study of Digital Storytelling is a fundamental part of understanding how digital games work in students' cognitive processes. This article is the result of a Systematic Review of the Literature followed by a meta-analysis of 28 studies that shows the correlation of the theory for the use of Digital Narrative along with Digital Game-based Learning. It is concluded that future studies respond as the Digital Narrative applied in educational digital games infer in the reflection of the subject and can contribute to that in order to obtain the learning process in an effective form.
\end{abstract}

KEYWORDS: Digital Storytelling. Digital Games-Based Learning. Digital Games.

\section{RESUMEN}

El uso de juegos digitales como apoyo a los procesos de enseñanza y aprendizaje ha sido un campo de investigación en pleno desarrollo. $Y$ el estudio de Digital Storytelling es una parte fundamental de la comprensión de cómo los juegos digitales actúan sobre los procesos cognitivos de los estudiantes. Este artículo es el resultado de una revisión sistemática de la literatura que resulta en un meta-análisis de 28 estudios que correlacionan el uso de la teoría Digital Storytelling com la Aprendizaje Basado em Juegos Digitales. Llegamos a la conclusión de que los estudios futuros responden como Digital Storytelling aplica en los juegos digitales educativos infieren el tema de reflexión y contribuyen a conseguir el mismo aprendizaje eficaz.

PALABRAS CLAVE: Digital Storytelling. Aprandizaje Basado em Juegos Digitales. Juegos Digitales.

\footnotetext{
${ }^{1}$ Doutorando em Jornalismo - Universidade Federal de Santa Catarina (UFSC) - Florianópolis, SC - Brasil. E-mail: ricardoaoki13@gmail.com

${ }^{2}$ Doutora em Psicologia - Universidade Federal do Rio Grande do Sul (UFRGS) - Porto Alegre, RS - Brasil.

Professora Programa de Pós-graduação em TIC - Universidade Federal de Santa Catarina (UFSC) -

Florianópolis, SC - Brasil. E-mail: pjfiuza@yahoo.com.br

${ }^{3}$ Doutor em Ciência da Computação - University of Calgary, Canadá. Professor Adjunto DE - Universidade Federal de Santa Catarina (UFSC) - Florianópolis, SC - Brasil. E-mail: robson.r.lemos@gmail.com
}

Submetido em: 18/07/2017 - Aceito em: 20/08/2018

(C) ETD-Educação Temática Digital Campinas, SP $\quad$ v.20 n.4 p.1117-1160 out./dez. 2018 


\section{INTRODUÇÃO}

É fato que a indústria dos jogos digitais tem crescido vertiginosamente nos últimos anos e a influência desse ambiente computacional na sociedade tem causado diversas quebras de paradigmas. Segundo pesquisa da Newzoo de 2017, existem 66,3 milhões de gamers no Brasil e uma movimentação de consumo de jogos digitais de US\$ 1,3 bilhão no mesmo ano o que faz o Brasil ser o principal mercado de jogos da América Latina e o décimo terceiro no ranking mundial (NEWZOO, 2017).

Os jogos de videogame deixaram a percepção, como se imaginava no início, de serem um passatempo dispersivo e aos poucos ganharam importância cultural na sociedade moderna. O segmento de jogos digitais pode ser considerado uma das atividades mais dinâmicas da economia criativa. Nesse sentido o seu processo produtivo se apropria da criatividade para a criação de um valor de mercado (LIMA, 2016). Sua influência nos processos de aprendizagem e o impacto que causam na educação ainda não são totalmente entendidos. Entretanto, um número crescente de pesquisadores estuda o impacto que essa poderosa mídia computacional tem exercido sobre professores, estudantes e sistemas educacionais.

Aprendizagem Baseada em Jogos Digitais (ABJD) tem se tornado um amplo campo de pesquisas. Para Prensky (2012, p. 22), "bem antes de os adolescentes de hoje terem netos, a aprendizagem baseada em jogos digitais - ou, precisamente, seus sucessores bem mais sofisticados - será considerada uma forma de aprender bastante normal". Esse efeito previsto por Prensky já pode ser notado em plataformas de aprendizagem digitais populares como Duolingo (Idiomas), Khan Academy (matemática, ciência, programação de computadores, história, história da arte, economia, etc) ou Codecademy (programação). Nesses Ambientes Virtuais de Aprendizagem (AVAs) os conceitos são aplicados de forma tão sutil que, muitas vezes, o usuário não percebe que está aprendendo e jogando.

O aprendizado tradicional, onde o professor está sempre narrando o seu conhecimento para os estudantes, não está propriamente com seus dias contados. Ele está em transformação. Quando crianças pequenas entram na escola elas são apresentadas formalmente para as narrativas tradicionais de aprendizado (MADEJ, 2007). Na ABJD a forma como o aprendizado é apresentado também se faz por um processo narrativo onde o aprendiz percebe rapidamente quando uma mídia se trata de um suporte de aprendiazado. Para Madej (2007) a narrativa em jogos digitais permite que o jogador tenha um sentimento de pertencimento com o conteúdo. Para a autora os jogos digitais oferecem oportunidades para os aprendizes tomarem suas próprias decisões. Dessa forma, as recompensas se tornam uma atividade prazerosa e interativa onde o resultado é diferente das formas narrativas tradicionais de aprendizado.

(C) ETD-Educação Temática Digital Campinas, SP $\quad$ v.20 n.4 $\quad$ p.1117-1160 $\quad$ out./dez. 2018


O presente artigo foi concebido a partir de uma pesquisa bibliográfica da literatura para identificar o estado da arte sobre a utilização de narrativas digitais em ambientes de aprendizagem baseada em jogos digitais. O método utilizado foi a Revisão Sistemática da Literatura (RSL) que segundo Freire (2013, p.33) "absorve os resultados dos estudos de outros pesquisadores, tratando seus resultados como estudos secundários. Da RSL saem análises que demandam novos estudos que, por sua vez irão gerar novos estudos primários". Essa Revisão Sistemática da Literatura é importante para definir o estado da arte referente a utilização de narrativas digitais aplicadas em ABJD, pois os pesquisadores da área poderão, a partir dela, identificar estudos atualizados em uma bibliometria da literatura que correlaciona as teorias correlacionadas em uma meta análise sobre os temas. Possibilitando, a partir de então, que se possa avançar na compreensão de uma área de estudos em crescimento. Esse levantamento foi realizado de agosto a novembro de 2016 e atualizado em novembro de 2017 e os resultados dessa pesquisa são apresentados a seguir.

\section{APRENDIZAGEM BASEADA EM JOGOS DIGITAIS}

Apesar do primeiro jogo eletrônico ter surgido por volta de 1951 e existirem pesquisas desde as décadas de 70-80 do século XX sobre como o uso de jogos digitais pode fornecer desafios e metas para os processos de ensino-aprendizagem (NETTO, 2014), foi somente a partir de 2001 que o termo Aprendizagem Baseada em Jogos Digitais (ABJD) foi popularizado por pesquisadores norte-americanos como Mark Prensky e por J. Paul Gee a partir de 2007 (LITTLE, 2015). No Brasil, segundo Alves (2008, p.227) "as discussões acerca da presença das tecnologias no cenário pedagógico já trilham um caminho de mais de vinte anos". Entretanto, até agora existe certo preconceito com relação aos jogos digitais no cenário pedagógico. Nesse sentido Alves (2008), das mais antigas pesquisadoras dos jogos digitais no cenário educacional brasileiro, relata que ainda existe desconfiança no potencial pedagógico desses elementos que ainda são vistos de forma maniqueísta pelos pares da área.

Brom, Šisler e Slavík (2009, p. 24, tradução nossa) também defendem posição parecida quando dizem que "entre a maioria dos professores e também muitos estudantes; jogos tendem a ser percebidos como uma atividade de lazer sem valor pedagógico, exceto para o desenvolvimento de competências em TI". Dessa forma é preciso avançar em direção de fomentar pesquisas em ABJD que orientem e ampliem a quebra de paradigma com relação a introdução dessa nova forma de aprendizado nas escolas como objeto de apoio ao aprendizado. Como defende Prensky (2012, p. 37) ao dizer que é necessário a "atualização do treinamento e do sistema educacional para que estes se tornem mais eficazes e eficientes."

Outro fator importante que necessita esclarecimento quando se trata especificamente de jogos digitais no ambiente educacional é a ideia que se está gamificando
(C) ETD- Educação Temática Digital
Campinas, SP
v.20 n. 4
p.1117-1160
out./dez. 2018 
o aprendizado. O termo gamificar vem sendo utilizado para classificar diversas áreas que estudam os jogos digitais. Entretanto, ele está relacionado com a utilização de conceitos de jogos em situações de não jogo (LEE, 2015) e a aprendizagem baseada em jogos digitais explora como os jogos com resultados de aprendizagem definidos, contribuem para a pedagogia (PRENSKY 2015 apud LEE, 2015). Isso quer dizer que muitos softwares educacionais são gamificados, ou seja, utilizam ideias provindas dos jogos digitais para avaliar ou elucidar os conteúdos aplicados aos estudantes. Todavia, a ABJD não pretende delegar aos jogos digitais o papel que é do professor e sim ser um objeto de apoio que utilize a gamificação em prol do aprendizado.

\section{NARRRATIVA DIGITAL}

O aprendizado pressupõe que o aprendiz tenha algum tipo de narração do conhecimento para que consiga processar mentalmente a informação recebida. Freire $(1987$, p.33) relata que no sistema capitalista as relações educador-educandos, na escola, em qualquer de seus níveis, apresentam um caráter especial e marcante, o de serem relações fundamentalmente narradoras. Entretanto, o autor defende que o papel do professor não pode se limitar a contação de histórias sobre determinado assunto. Para Bell (2014) a habilidade da narração, em tempos de mídias digitais, deve ser incorporada no aprendizado, pois narrar histórias são a nossa forma de comunicação a qual criamos nossos filhos e essa habilidade deveria ser incorporada na aprendizagem.

O método tradicional de ensino utiliza a contação de histórias (Storytelling) e as narrativas desde os primórdios. Esse método é tão ou mais antigo que a própria linguagem na comunicação humana. Freire $(1987$, p. 33) afirma que isso tende a petrificar ou a fazer-se algo quase morto e que essa narração implica num sujeito - o narrador - e em objetos pacientes, ouvintes - os educandos. Atualmente, mesmo que o aluno queira aprender por conta própria, este, necessitará quase que obrigatoriamente, ter contato com mídias, analógicas ou digitais, que the roteirizem algum tipo de caminho a ser seguido. E a busca pela inserção das Tecnologias da Informação e Comunicação (TIC) na aprendizagem se faz com a necessidade de entender os processos narrativos que estão inseridos nas interfaces dos dispositivos computacionais. Entretanto, essa nova maneira de ensinar, através das TIC, ainda é um paradigma que para Lévy (2010, p.05) está longe de ser alcançado:

É certo que a escola é uma instituição que há cinco mil anos se baseia no falar/ditar do mestre, na escrita manuscrita do aluno e, há quatro séculos, em um uso moderado da impressão. Uma verdadeira integração da informática (como do audiovisual) supõe, portanto, o abandono de um hábito antropológico mais que milenar, o que não pode ser feito em alguns anos.

Assim sendo surgem novos estudos que tentam entender a aplicação de narrativas digitais como método de aprendizagem. Em especial os jogos sérios que utilizam esse método na Aprendizagem Baseada em Jogos Digitais. Porém, é necessário distinguir o termo
(C) ETD- Educação Temática Digital
Campinas, SP
v. 20 n. 4
p.1117-1160
out./dez. 2018 
de forma correta. Existem duas terminologias a serem pensadas quando falamos de narrativa digital em jogos digitais e que se confundem pelas peculiaridades da Língua Portuguesa e que são bem definidas em Língua Inglesa. Neste artigo, quando falarmos em Narrativa Digital, estamos nos referindo ao termo em inglês Digital Storytelling, que é definido por Rolling e Adams (2003, p. 10, apud Swan, 2009, p. 55-56, tradução nossa) "como um problema não resolvido, ou conflito que mantém a atenção do leitor". Sendo assim, sugerimos ao leitor que pense em Narrativa Digital em um contexto narrativo sem regras pré-definidas de começo, meio e fim. $\mathrm{O}$ autor ainda afirma que nem todos os jogos digitais possuem uma narrativa. Entretanto o elemento Storytelling sobre a tensão exercida sempre estará presente.

Essa distinção é necessária para que o objeto de pesquisa seja tratado de forma correta. Neste artigo sempre utilizaremos os termos em português. Sendo assim, a narrativa estará ligada ao enredo da história e a Narrativa Digital às questões de atenção do jogador e como o mesmo é "fisgado" pelo enredo. Porém, não será incomum encontrar os termos misturados ou ora utilizados por um autor de uma forma diferente da entendida por Rolling e Adams. Para Reeves e Read (2009, apud Jenkins, 2011) o Storytelling é a melhor forma para transmitir informações, porque o conteúdo é mais facilmente lembrado se for entregue em um contexto narrativo. Os autores defendem não ser possível apenas narrar os fatos. É preciso coloca-los em um contexto sequenciado de início meio e fim e considerar a tensão sobre como o enredo do jogo digital vai se resolver.

Dessa forma ao utilizar a Aprendizagem Baseada em Jogos Digitais se faz necessário entender a abordagem narrativa que estará sendo aplicada no processo de aprendizagem. Sabendo que a narrativa, por si só, não é um elemento presente em todos os jogos digitais, o desafio é filtrar as terminologias apresentadas por diversos autores quando se trata de narrativas digitais. Assim será possível entender como cada estudo tratou do tema já que nas análises preliminares dessa pesquisa, se identificou que a diferença entre narrativa e narrativa digital não é consensual.

\section{METODOLOGIA}

A pesquisa foi elaborada a partir da necessidade de identificar estudos sobre a utilização de Narrativas Digitais na Aprendizagem Baseada em Jogos Digitais e como essas duas teorias se relacionam. Para tal, foi adotada uma busca exploratória através de uma Revisão Sistemática da Literatura (RSL). Esse método foi escolhido para que se possa identificar uma síntese do conhecimento já adquirido sobre o tema (VELLUZZI, 1998 apud FREIRE 2013, p.31) e assim possibilitar caminhos para identificar possíveis avanços ao se comparar e estatisticamente relacionar os resultados de diferentes pesquisas que levem a novos resultados (FREIRE, 2013, p.28).

\footnotetext{
(C) ETD-Educação Temática Digital Campinas, SP $\quad$ v.20 n.4 $\quad$ p.1117-1160 out./dez. 2018
} 
Questão de Pesquisa

Todo trabalho acadêmico requer uma pesquisa bibliográfica para se explicar um problema. Rampazzo (2013, p.53) pressupõe "que qualquer espécie de pesquisa, em qualquer área, supõe e exige uma pesquisa bibliográfica prévia". Dessa forma será possível entender a questão que se pretende responder. Galvão e Pereira (2014, p.183) definem que uma "revisão sistemática da literatura. Trata-se de um tipo de investigação focada em questão bem definida, que visa identificar, selecionar, avaliar e sintetizar as evidências relevantes disponíveis". Sendo assim o primeiro passo para este estudo foi formular a pergunta sobre o problema ao qual se pretende responder.

Gomes e Caminha (2014, p.402) orientam que o "primeiro passo a ser dado no início de qualquer estudo é estabelecer o que se deseja pesquisar. Questões mal formuladas podem conduzir a decisões obscuras sobre o que incluir na revisão posteriormente". Corroborando com Galvão e Pereira (2014, p.184) ao orientarem que "uma boa pergunta de pesquisa analítica, a que investiga a relação entre dois eventos, é formada por diversos componentes". Cabendo ao pesquisador definir os critérios de temporalidade, público alvo e teorias aos quais deseja despender tempo e esforço no seu trabalho.

Assim sendo o pesquisador definiu que a teoria a ser estudada é a Aprendizagem Baseada em Jogos Digitais com o recorte específico de entender a utilização da Narrativa Digital em jogos digitais educativos. E a pergunta de pesquisa definida foi: Qual a utilização da Narrativa Digital em Ambientes de Aprendizagem Baseada em Jogos Digitais?

\section{Protocolo da Revisão Sistemática da Literatura}

Para a aplicação da pesquisa foram definidas as seguintes variáveis, e suas variantes em Língua Inglesa, de busca nas bases de dados e no motor de busca: Variável 1: Aprendizagem Baseada em Jogos Digitais (Digital Game-Based Learning) e a Variável 2: Narrativa Digital (Digital Storytelling). Também foram definidas as seguintes palavras chaves, e suas variantes em Língua Inglesa, no auxílio do enquadramento dos documentos a serem pesquisados: Aprendizagem (Learning), Educação (Education), Jogos (Games), Narrativa (Storytelling, Narrative).

A seleção das bases de dados para a pesquisa levou em consideração a disponibilidade de acesso aos artigos, dissertações e teses. Por isso, foram priorizadas as plataformas conveniadas com o Portal de Periódicos da Capes através do IP da Universidade Federal de Santa Catarina. A busca foi realizada em Língua Portuguesa e Inglesa em todas as bases de dados conforme o Quadro 1 a seguir:

\footnotetext{
(C) ETD- Educação Temática Digital Campinas, SP $\quad$ v.20 n.4 $\quad$ p.1117-1160 out./dez. 2018
} 
DOI: 10.20396/etd.v20i4.8649913

QUADRO 1 - Bases de dados utilizadas para consulta e parâmetros de busca.

\begin{tabular}{|c|c|}
\hline $\begin{array}{l}\text { Base de Dados/Motor de } \\
\text { Busca }\end{array}$ & Parâmetros de busca \\
\hline Scopus Elsevier & (TITLE-ABS-KEY(Digital Game-Based Learning)) AND (storytelling) \\
\hline Web Of Science & $\begin{array}{c}\text { (Digital Games-Based Learning) } \\
\text { Refinado por: Tópico: (Digital Storytelling) } \\
\text { Tempo estipulado: Todos os anos. Índices: SCl-EXPANDED, SSCI, A\&HCI, CPCI-S, } \\
\text { CPCI-SSH, ESCl. }\end{array}$ \\
\hline Proquest & $\begin{array}{c}\text { ("digital game-based learning" storytelling) AND subt.exact("educational } \\
\text { technology" OR "educational software") }\end{array}$ \\
\hline $\begin{array}{l}\text { Google Schoolar } \\
\text { (Motor de Buscas) }\end{array}$ & $\begin{array}{l}\text { Pesquisa em Inglês: } \\
\text { "Digital Game-Based Learning" "Digital Storytelling" } \\
\text { Últimos } 10 \text { anos, sem patentes e citações } \\
\text { Pesquisas nas páginas em Língua Portuguesa } \\
\text { "Aprendizagem Baseada em Jogos Digitais"'”Narrativa Digital" } \\
\text { Últimos } 10 \text { anos, sem patentes e citações }\end{array}$ \\
\hline
\end{tabular}

Para a busca foram considerados os seguintes critérios de inclusão e exclusão de documentos.

QUADRO 2 - Critérios de Busca e Exclusão.

\begin{tabular}{|c|c|c|}
\hline Fase & Critérios de Inclusão para análise & Critérios de Exclusão para análise \\
\hline \multirow{7}{*}{$\begin{array}{c}\text { Seleção e identificação dos } \\
\text { Estudos }\end{array}$} & Artigo completo & Artigo Pago \\
\hline & Base de dados com acesso livre & Artigos de Conferências \\
\hline & Revisado por pares & $\begin{array}{l}\text { Trabalho de Conclusão de Curso } \\
\text { (Graduação) }\end{array}$ \\
\hline & Publicado em Português ou Inglês & $\begin{array}{c}\text { Trabalhos em outros idiomas que } \\
\text { não o Português e o Inglês }\end{array}$ \\
\hline & $\begin{array}{l}\text { Descritores no título, resumo ou } \\
\text { palavras chave }\end{array}$ & Documentos com mais de 10 anos \\
\hline & & $\begin{array}{c}\text { Não constar os descritores ou } \\
\text { derivações no Título, Resumo ou } \\
\text { Palavras Chave }\end{array}$ \\
\hline & & $\begin{array}{c}\text { Após a leitura do resumo } \\
\text { identificar que o assunto não } \\
\text { relaciona as variáveis pesquisadas }\end{array}$ \\
\hline Extração dos Dados & & $\begin{array}{l}\text { Após a leitura do documento } \\
\text { identificar que o assunto não tem } \\
\text { relação com a pesquisa }\end{array}$ \\
\hline
\end{tabular}

(C) ETD-Educação Temática Digital Campinas, SP $\quad$ v.20 n.4 p.1117-1160 out./dez. 2018 
Também foram definidos uma pontuação no software de extração de dados com a finalidade de criar uma pontuação (em inglês score) qualitativa para auxiliar na seleção dos estudos. Esse score levou em consideração a quantidade de vezes que as palavras definidas nas variáveis 1 e 2 aparecem no título, resumo e palavras chaves dos documentos a serem analisados. A pontuação aplicada foi de cinco pontos para cada ocorrência no título, três pontos para o resumo e dois pontos nas palavras chaves.

\section{SELEÇÃO DOS ESTUDOS}

A seleção de estudos sobre utilização de Narrativas Digitais em ambientes de Aprendizado Baseado em Jogos Digitais se divide em identificar os documentos e posteriormente extrair as informações dos mesmos para a realização de uma metanálise. A Revisão Sistemática da Literatura pressupõe que os métodos utilizados para tal trabalho devem ser claros para que outros pesquisadores possam reproduzir o estudo. Sendo assim, essa seção detalha os procedimentos utilizados para a seleção dos documentos utilizados na presente análise.

O primeiro passo foi inserir a variável 1 nas bases de dados e motor de busca como critério inicial de seleção de documentos. Após o primeiro resultado a variável 2 foi inserida para refinar os resultados. No QUADRO 1 é possível verificar os critérios utilizados para refinar os parâmetros de busca. O parâmetro de cada base de dados se utilizado da mesma forma em pesquisas futuras devem, em princípio, retornar os mesmos resultados do presente estudo.

Ao final das buscas nas bases de dados e no motor de busca os pesquisadores identificaram 504 estudos que relacionam as variáveis definidas no protocolo desta Revisão Sistemática da Literatura conforme a TABELA 1.

TABELA 1 - Resultados da busca nas bases de dados e no Motor de Busca.

\begin{tabular}{ccc}
\hline Base de dados ou Motor de Busca & $\begin{array}{c}\text { Resultado inicial } \\
\text { Variável 1 } \\
\text { (Narrativa Digital) }\end{array}$ & $\begin{array}{c}\text { Resultado refinado } \\
\text { Variável } 2 \\
\text { (ABJD) }\end{array}$ \\
\hline $\begin{array}{c}\text { Google Acadêmicos (Busca em Inglês) } \\
\text { Google Acadêmicos (Busca em Português) } \\
\text { Scopus Elsevier }\end{array}$ & 15400 & 249 \\
Web of Science & 12500 & 60 \\
Proquest & 880 & 16 \\
\hline Total & 453 & 98 \\
\hline
\end{tabular}

(C) ETD-Educação Temática Digital Campinas, SP $\quad$ v.20 n.4 $\quad$ p.1117-1160 out./dez. 2018 
A partir dessa etapa os pesquisadores utilizaram a ferramenta Start - State of the Art through Systematic Review ${ }^{4}$ desenvolvida pelo Laboratório de Pesquisa em Engenharia de Software da Universidade Federal de São Carlos. O software é livre e gratuito e foi desenvolvido para auxiliar pesquisadores no processo de mensuração dos dados em uma Revisão Sistemática de Literatura.

A seleção dos documentos teve como critérios de inclusão e exclusão os argumentos listados na QUADRO 2 do Protocolo de RSL. O primeiro passo foi utilizar a ferramenta Start para auxiliar na identificação de documentos duplicados nas bases de dados. Nesse processo surgiram 21 duplicações. Reduzindo o número de documentos identificados ao total de 468. Após esse passo, aplicou-se um filtro para identificar os documentos provenientes de anais de eventos, que conforme os critérios do protocolo seriam rejeitados da seleção. Com esse filtro a ferramenta Start encontrou mais 155 documentos que foram subtraídos da análise. A partir desse momento foi dado início ao processo de leitura de títulos, resumos e palavras chaves dos 313 documentos restantes, a fim de identificar, numa primeira impressão, todos que relacionem diretamente a utilização de Narrativas Digitais em Ambiente de Aprendizado Baseado em Jogos Digitais.

Durante o processo de leitura foram identificados mais 25 documentos em bases de dados onde o acesso é pago ou restrito mesmo acessando pelo IP institucional da UFSC. De 288 artigos com resumos, títulos e palavras chaves lidos, foram rejeitados 241 pela avaliação que, ou discorriam apenas sobre algum tipo de Narrativa Digital sem relação com a Aprendizagem Baseada em Jogos Digitais ou que tratavam superficialmente sobre algum aspecto das duas teorias em estudo. Dessa forma restaram 47 artigos para a fase de extração de dados que descrevemos a seguir:

\section{Extração de dados}

Segundo Pereira e Galvão (2014, n.p.) se deve definir previamente quais informações serão absorvidas dos documentos. Para os autores "em geral, coletam-se dados sobre autor, data de publicação, tipo de estudo, participantes (número, média de idade, proporção de cada sexo), exposição ou intervenção, desfecho, tempo de seguimento e perdas". Sendo assim foram definidos os seguintes critérios para extração de dados:

Foi definido que a leitura detalhada dos artigos deveria identificar os estudos que tenham relacionado a utilização de Narrativas Digitais com a Aprendizado Baseado em Jogos Digitais e que todos os documentos que tratassem apenas de uma teoria e não de outra seriam excluídos da extração. Se definiu que a teoria de aprendizagem, objetivo do estudo, tipo de estudo, metodologia de análise, vantagens e desvantagens, novas terminologias

\footnotetext{
${ }^{4} \mathrm{O}$ download do software Start pode ser feito em: http://lapes.dc.ufscar.br/tools/start_tool
}

(C) ETD- Educação Temática Digital Campinas, SP $\quad$ v.20 n.4 $\quad$ p.1117-1160 out./dez. 2018


para as teorias estudas, as conclusões e sugestões de estudos futuros deveriam ser observadas para a realização de bibliometria e metanálise posterior. Com relação ao software atentou-se para sua funcionalidade, tipo de software (se livre ou proprietário), faixa etária indicada e público alvo.

Com relação ao período de publicação dos artigos selecionados para extração, foi gerado a Figura 1 a qual demonstra uma concentração de publicações nos anos de 2014 e 2015. Os estudos que relacionam as duas variáveis de pesquisa, se mantém na média até 2013 e tem uma elevação em 2014 e 2015 e voltando a média em 2016. Com relação ao ano de 2017, até a data de atualização da RSL, não se identificou novos estudos relevantes que correlacionam às variáveis discutidas nessa pesquisa.

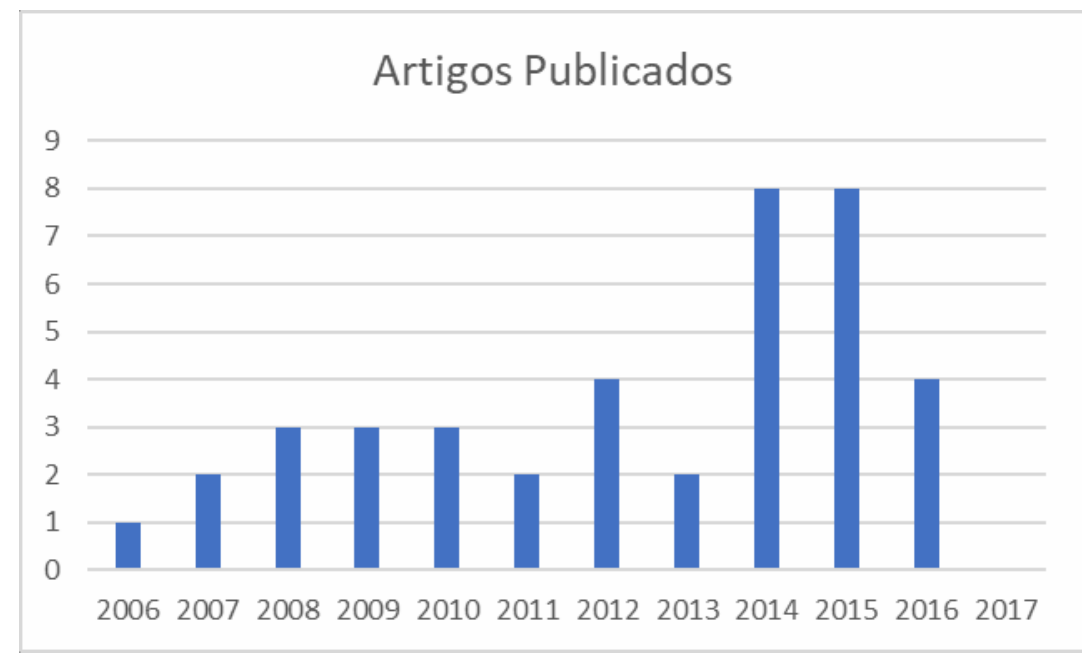

FIGURA 1 - Artigos publicados.

Fonte: Os autores

Após a leitura completa dos 47 documentos selecionados na fase da extração. Foram excluídos da análise um documento duplicado que a ferramenta Start não conseguiu identificar ao aplicar o filtro anteriormente, isso por causa de um erro ortográfico que diferenciavam o documento em duas bases de dados distintas. Outros dezoito documentos também foram rejeitados por não atenderem os critérios para a extração de dados. Nessa fase da presente revisão sistemática foram selecionados 28 documentos para a sumarização dos dados. O Quadro 3 relaciona os documentos. 
DOI: 10.20396/etd.v20i4.8649913

QUADRO 3 - Bibliometria dos documentos selecionados na extração de dados.

\begin{tabular}{|c|c|c|c|c|c|}
\hline Referência & Pontuação & Ano & Tipo & Base de Dados & Citações \\
\hline Hsiao (2007) & 355 & 2007 & Tese ou Dissertação & Proquest & 4 \\
\hline Madej (2007) & 275 & 2007 & Tese ou Dissertação & Proquest & 4 \\
\hline Brom, Sisler e Slavik (2009) & 245 & 2009 & Artigo & Scopus & 83 \\
\hline Tomaso $(2014)$ & 215 & 2014 & Tese ou Dissertação & Proquest & 2 \\
\hline Padilla-Zea et al. (2014) & 160 & 2014 & Artigo & Scopus & 24 \\
\hline Neville (2010) & 155 & 2010 & Artigo & Scopus & 5 \\
\hline Harrold (2015) & 150 & 2015 & Tese ou Dissertação & Proquest & 1 \\
\hline Rodrigues e Bidarra (2014) & 130 & 2014 & Artigo & Scopus & 0 \\
\hline Yang e Chang (2013) & 120 & 2013 & Artigo & Scopus & 29 \\
\hline Swan (2008) & 120 & 2008 & Tese ou Dissertação & Proquest & 4 \\
\hline Fooks (2014) & 115 & 2014 & Tese ou Dissertação & Proquest & 2 \\
\hline Renner (2013) & 100 & 2014 & Tese ou Dissertação & Proquest & 1 \\
\hline Ferguson (2014) & 100 & 2014 & Tese ou Dissertação & Proquest & 7 \\
\hline WEIß e Muller (2008) & 95 & 2008 & Artigo & Scopus & 1 \\
\hline Rubino et al. (2015) & 90 & 2015 & Artigo & Scopus & 11 \\
\hline Lee (2015) & 75 & 2015 & Tese ou Dissertação & Proquest & 0 \\
\hline Augustin et al. (2013) & 70 & 2013 & Artigo & Scopus & 8 \\
\hline Bell (2014) & 60 & 2014 & Tese ou Dissertação & Proquest & 0 \\
\hline Kronenberg (2013) & 30 & 2013 & Capítulo de Livro & Google Schoolar (EN) & 2 \\
\hline Coelho e Costa (2016) & 15 & 2016 & Artigo & Google Schoolar (PT) & 2 \\
\hline Silva (2014) & 15 & 2014 & Tese ou Dissertação & Google Schoolar (PT) & 0 \\
\hline Vidani (2012) & 15 & 2012 & Tese ou Dissertação & Google Schoolar (EN) & 0 \\
\hline Bellotti, Berta e Gloria (2010) & 15 & 2010 & Artigo & Google Schoolar (EN) & 98 \\
\hline Park (2012) & 15 & 2012 & Artigo & Google Schoolar (EN) & 34 \\
\hline Dominguez et al. (2015) & 0 & 2015 & Artigo & Google Schoolar (PT) & 2 \\
\hline Guerreiro (2015) & 0 & 2015 & Tese ou Dissertação & Google Schoolar (PT) & 2 \\
\hline Sousa (2015) & 0 & 2015 & Tese ou Dissertação & Google Schoolar (PT) & 0 \\
\hline Bordini (2016) & 0 & 2016 & Tese ou DissertaçãoTHES & Google Schoolar (PT) & 0 \\
\hline
\end{tabular}

No Quadro 3 os documentos foram classificados pelo score que demonstrou que Hsiao (2007) obteve a maior pontuação de acordo com os critérios definidos no protocolo da RSL. Na Figura 3 é possível verificar os gráficos que representam a porcentagem de artigos de acordo com o editor, bases de dados, tipos de documento e ano de publicação.
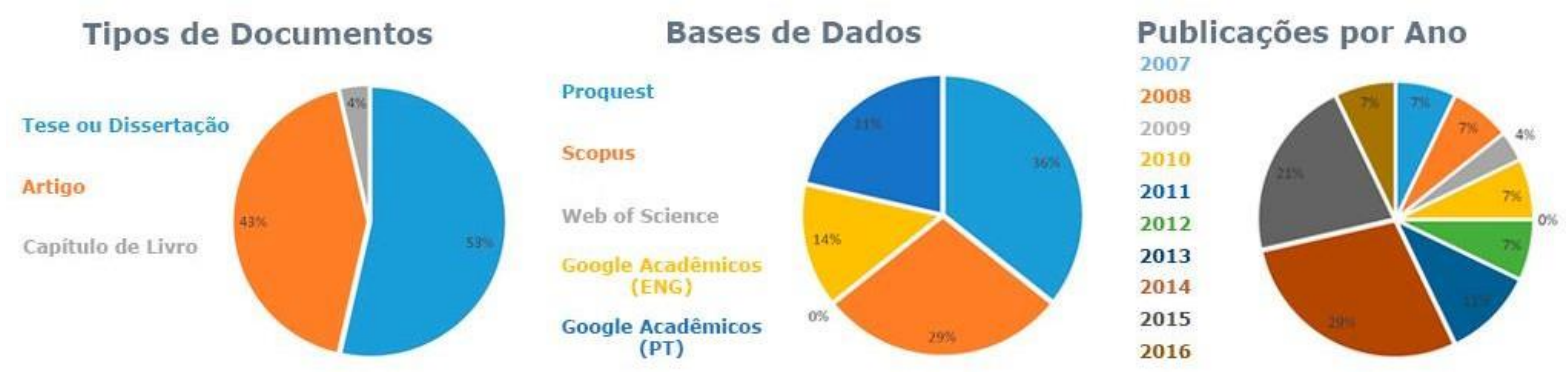

FIGURA 3 - Gráficos resultantes da bibliometria.

Fonte: Autores

(C) ETD- Educação Temática Digital Campinas, SP $\quad$ v.20 n.4 $\quad$ p.1117-1160 $\quad$ out./dez. 2018 
A relevância dos documentos foi determinada pelo número de citações. Nesse quesito o artigo de Bellotti, Berta e Glória (2010) se destacou com 39 citações. Na Figura 4 é possível verificar o gráfico que demonstra os artigos com mais citações.

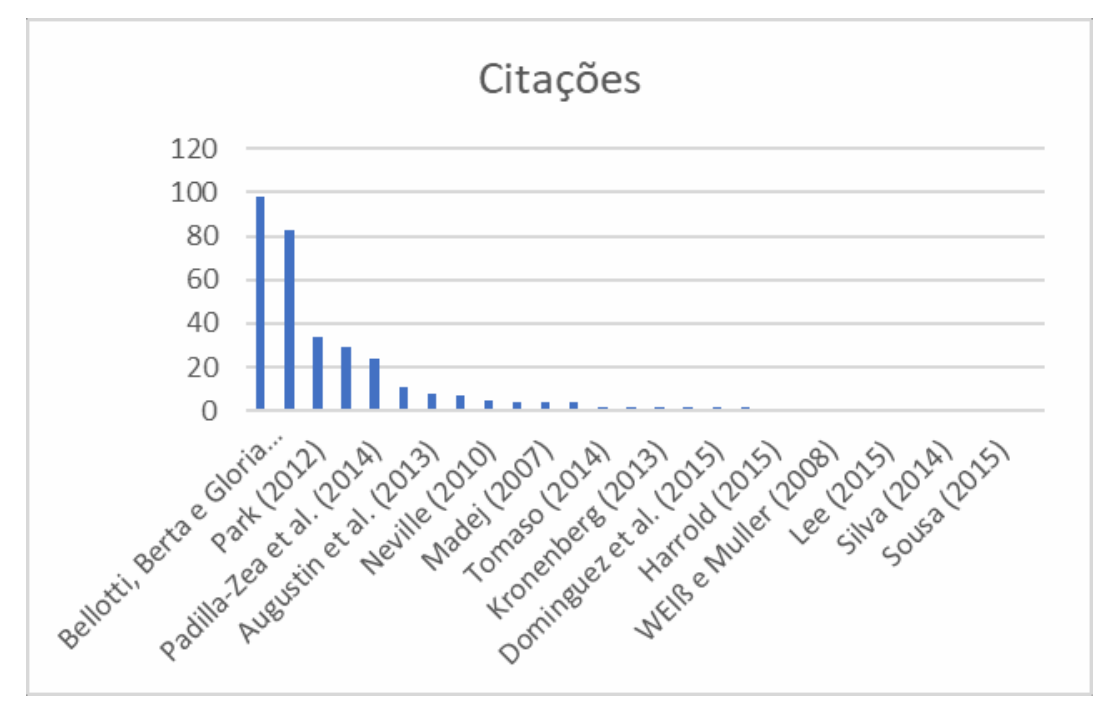

Figura 4 - Gráfico de número de citações.

Fonte: Autores

O Quadro 4 faz a bibliometria das teorias de aprendizagem que os autores utilizaram para relacionar a teoria da Narrativa Digital com a Aprendizagem Baseada em Jogos Digitais. Existe uma predominância pela teoria construtivista mencionada em sete documentos (Yang e Chang (2013), Renner (2013), Ferguson (2014), Rubino et al. (2015), Kronenberg (2013), Park (2012) e Dominguez et al. (2015)). No tocante às metodologias de análise, houve uma grande quantidade de métodos utilizados o que também ocorre nos tipos de estudo realizados. Dessa forma indicamos que pesquisadores interessados devem avaliar cada estudo individualmente. 
DOI: 10.20396/etd.v20i4.8649913

QUADRO 4 - Bibliometria sobre teorias de aprendizagem, métodos e tipos de estudo

\begin{tabular}{|c|c|c|c|}
\hline Referência & Teoria de & Metodologia da Análise & Tipo de estudo \\
\hline Hsiao (2007) & Aprendizagem & Entrevistas feitas por email & Estudo de caso \\
\hline Madej (2007) & Pedagogia Feminista & Qualitativa não positivista & Naturalista \\
\hline Brom, Sisler e Slavik & Centrada no Aluno & Pré testes e pós testes & Entrevistas em \\
\hline Tomaso (2014) & Multimodal & ANOVA multivariada & Teste de inventário \\
\hline Padilla-Zea et al. (2014) & Não identificado & Análise de Emoções & Aprendizado-jogo- \\
\hline Neville (2010) & Baseada em Contexto & Análise Conceitual & Ensaio \\
\hline Harrold (2015) & Aprendizagem & Entrevista Qualitativa & Estudo de Caso \\
\hline Rodrigues e Bidarra & Centrada no Aluno & Não foi possível identificar & Experimental e \\
\hline Yang e Chang (2013) & Construtivista & Análise Multivariada & Quasi-experimental \\
\hline Swan (2008) & Não identificado & Transportabilidade & Pesquisa exploratória \\
\hline Fooks (2014) & Inteligências Múltiplas & $\begin{array}{c}\text { Sistema de gerenciamento de } \\
\text { aprendizagem }\end{array}$ & $\begin{array}{l}\text { Experimental } \\
\text { quantitativa }\end{array}$ \\
\hline Renner (2013) & Construtivista & Pré-teste e pós-teste & Quasi-experimental \\
\hline Ferguson (2014) & Construtivista & Estatística descritiva & Comparativa de causa \\
\hline WEIß e Muller (2008) & Não identificado & Não foi possível identificar & Comparativo \\
\hline Rubino et al. (2015) & Construtivista & Entrevista semi-estruturada & Qualitativo \\
\hline Lee $(2015)$ & Não identificado & Entrevista estruturada & Observatória \\
\hline Augustin et al. (2013) & Baseado em & Avaliação probabilística & Simulação \\
\hline Bell (2014) & Não identificado & Entrevista Multivariada & Fenomenológica \\
\hline Kronenberg (2013) & Construtivista & Análise do estado da arte & Estado da Arte \\
\hline Coelho e Costa (2016) & Não identificado & Observação sistemática & Fenomenológica \\
\hline Silva (2014) & Sócio Histórica & Entrevista estruturada & Pesquisa qualitativa \\
\hline Vidani (2012) & Não identificado & Entrevista & Estudo de caso \\
\hline Bellotti, Berta e Gloria & Behaviorismo & Análise crítica quantitativa & Estado da Arte \\
\hline Park (2012) & Construtivista & Motivação Intrínseca & Experimental \\
\hline Dominguez et al. & Construtivista & Entrevistas e estatística & Experimental \\
\hline Guerreiro (2015) & Não identificado & Análise Mista & Pesquisa qualitativa \\
\hline Sousa (2015) & Sócio Interacionista & Entrevista semiestruturada & Pesquisa qualitativa \\
\hline Bordini (2016) & Não identificado & Entrevista semi estruturada & Observatória \\
\hline
\end{tabular}

No Quadro 5 constam as informações demográficas e a área de conhecimento de cada documento, país, área de estudos e população. Fica evidente uma clara predominância de pesquisas realizadas nos Estados Unidos, provavelmente por ser o país onde a tecnologia nasceu. Ou seja, um total de 11 referências. Mesmo número de referências relacionadas aos estudos na área da Educação (Figura 5) que também são predominantes. 
QUADRO 5 - Bibliometria sobre área de conhecimento e informações demográficas.

\begin{tabular}{|c|c|c|c|c|}
\hline Referência & País & Area de Conhecimento & Faixa Etária & Nível Escolar \\
\hline Hsiao (2007) & EUA & Educação Artística & Adultos & Não identificado \\
\hline Madej (2007) & Canadá & Artes e Tecnologia & Crianças até 7 anos & Não identificado \\
\hline $\begin{array}{l}\text { Brom, Sisler e } \\
\text { Slavik (2009) }\end{array}$ & $\begin{array}{c}\text { República } \\
\text { Checa }\end{array}$ & Sistemas Multimídia & Adolescentes & Ensino Médio \\
\hline Tomaso (2014) & EUA & Ciência da Computação & Não identificado & Ensino Médio \\
\hline Padilla-Zea et al. (2014) & Espanha & Engenharia de Software & Crianças até 7 anos & Ensino Básico \\
\hline Neville (2010) & EUA & Educação & Não identificado & Não identificado \\
\hline Harrold (2015) & EUA & Instrução Gestão e & Adolescentes & Ensino Médio \\
\hline $\begin{array}{c}\text { Rodrigues e } \\
\text { Bidarra (2014) }\end{array}$ & Portugal & Tecnologia Educacional & Crianças (11 a 13 anos) & Ensino Médio \\
\hline Yang e Chang (2013) & Taiwan & Educação & Crianças (11 a 13 anos) & Ensino \\
\hline Swan (2008) & EUA & Psic. e Tec. Educacional & Não identificado & Não identificado \\
\hline Fooks (2014) & EUA & Educação & Adultos & Ensino Superior \\
\hline Renner (2013) & EUA & Educação & Adolescentes & Ensino Médio \\
\hline Ferguson (2014) & EUA & Educação & Adolescentes & Ensino Médio \\
\hline WEIß e Muller (2008) & Alemanha & Pedagogia & Não identificado & Não identificado \\
\hline Rubino et al. (2015) & Itália & Artes e Humanidades & Adolescentes & Não identificado \\
\hline Lee (2015) & EUA & Ciência da Computação & Adolescentes & Ensino Médio \\
\hline Augustin et al. (2013) & Austria & Ciência da Computação & Não identificado & Não identificado \\
\hline Bell (2014) & EUA & Educação & Adultos & Ensino Superior \\
\hline Kronenberg (2013) & EUA & Educação & Não identificado & Não identificado \\
\hline Coelho e Costa (2016) & Brasil & Educação & Adolescentes & Ensino Médio \\
\hline Silva (2014) & Brasil & Educação & Não identificado & Não identificado \\
\hline Vidani (2012) & Itália & Ciência da Computação & Adultos & Ensino \\
\hline $\begin{array}{l}\text { Bellotti, Berta e Gloria } \\
\text { (2010) }\end{array}$ & Itália & $\begin{array}{l}\text { Ciências Sociais } \\
\text { Engenharia }\end{array}$ & Não identificado & Não identificado \\
\hline Park (2012) & Coreia do & Ciência da Computação & Crianças (11 a 13 anos) & Ensino \\
\hline Dominguez et al. (2015) & Brasil & Educação & Crianças (11 a 13 anos) & Ensino \\
\hline Guerreiro (2015) & Brasil & Educação para a ciência & Não identificado & Não identificado \\
\hline Sousa (2015) & Brasil & Educação Matemática e & Crianças (11 a 13 anos) & Ensino \\
\hline Bordini (2016) & Brasil & Música e Artes & Adultos & Ensino Superior \\
\hline
\end{tabular}

A Figura 5 mostra três gráficos que fazem uma breve análise da predominância das informações relacionadas no Quadro 5. Percebe-se que os documentos se relacionam predominantemente com a Educação e Ciência da Computação. Na faixa etária houve uma concentração em estudos que foram aplicados com adolescentes. Devido a isso, nota-se também que o Ensino Médio foi o ambiente mais utilizado para aplicar os testes. 

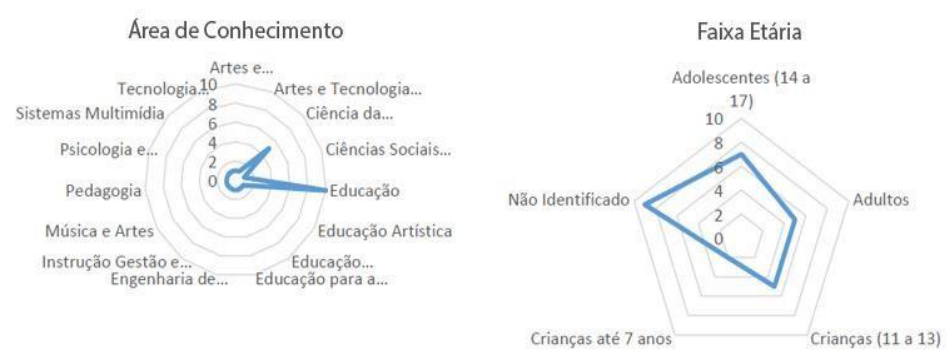

DOI: $10.20396 /$ etd.v20i4.8649913

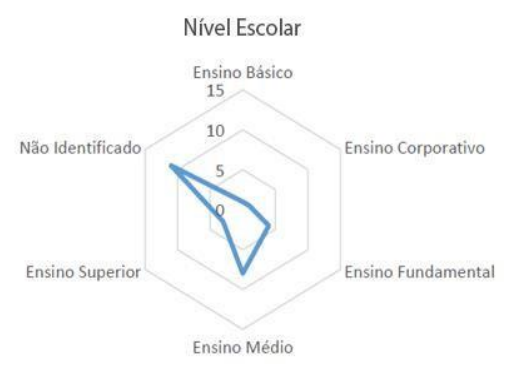

Figura 5 - Gráficos de dados demográficos.

Fonte: Autores

No Quadro 6 foram extraídos os dados relacionados com às Tecnologias da Informação e Comunicação (TIC). Nesse quadro estão os dispositivos tecnológicos utilizados para cada experimento e as informações referentes aos softwares e tipo de softwares aplicados aos estudos.

QUADRO 6 - Bibliometria referente às Tecnologias da Informação e Comunicação.

\begin{tabular}{|c|c|c|c|c|}
\hline Referência & Disp & Software & TIPO & Função \\
\hline Hsiao (2007) & $\mathrm{C}$ & The Sim's 2 & Prop & Simulador de vida \\
\hline Madej (2007) & C & CD Rom com jogos & Livre & Jogos Narrativos Interativos para crianças \\
\hline $\begin{array}{l}\text { Brom, Sisler e Slavik } \\
\text { (2009) }\end{array}$ & C & Europa 2045 & Livre & $\begin{array}{l}\text { Incentiva modelos mentais, Geografia } \\
\text { européia e Tomada de decisão }\end{array}$ \\
\hline Tomaso (2014) & C & FastMath & Livre & Ensino de matemática \\
\hline $\begin{array}{l}\text { Padilla-Zea et al. } \\
\text { (2014) }\end{array}$ & M & ‘Ato's Adventure & Livre & Treinamento de motricidade \\
\hline Neville (2010) & C & Não identificado & Livre & Ensino de segundo idioma através de 3d DGBL \\
\hline Harrold (2015) & $\mathrm{C}$ & 3d GameLab & Livre & Criação de jogos \\
\hline $\begin{array}{l}\text { Rodrigues e Bidarra } \\
\text { (2014) }\end{array}$ & C & Inanimated Alice & Prop & $\begin{array}{c}\text { Jogo colaborativo para criação de } \\
\text { enredos }\end{array}$ \\
\hline $\begin{array}{l}\text { Yang e Chang } \\
\text { (2013) }\end{array}$ & M & RPG Maker & Livre & Criação de jogos RPG \\
\hline Swan (2008) & $\mathrm{C}$ & Não identificado & Livre & Não identificado \\
\hline Fooks (2014) & C & $\begin{array}{c}\text { Blackboard Management } \\
\text { System }\end{array}$ & Prop & Ensino de Matemática \\
\hline Renner (2013) & M & Aris Game & Livre & $\begin{array}{c}\text { Ensino de Química com jogos de } \\
\text { Realidade Aumentada }\end{array}$ \\
\hline Ferguson (2014) & M & $\begin{array}{l}\text { Plataforma Quia } \\
\text { Shodor }\end{array}$ & Livre & Ensino de Algebra \\
\hline $\begin{array}{l}\text { WEIß e Muller } \\
\text { (2008) }\end{array}$ & C & Scenejo & Livre & Criação de Interactive Storytelling \\
\hline Rubino et al. (2015) & M & Gossip at palace & Livre & $\begin{array}{c}\text { Integração da mecânica do jogo e da } \\
\text { observação em museus }\end{array}$ \\
\hline Lee (2015) & $\mathrm{C}$ & Gidget & Livre & Ensinar programação de computadores \\
\hline Augustin et al. (2013) & C & Eight Days & Livre & Ensino de Geografia \\
\hline Bell (2014) & C & $\begin{array}{l}\text { The Fairy Tale MOOC } \\
\text { The Hero's Journey } \\
\text { Dungeons and Discourse } \\
\text { Ethical Decision Making }\end{array}$ & Livre & Jogos narrativos utilizados em AVAS \\
\hline Kronenberg (2013) & C & $\begin{array}{c}\text { Mingoville } \\
\text { PaRappa the Rapper } 2 \\
\text { Ausflug nach München }\end{array}$ & Livre & Ensino de linguas \\
\hline
\end{tabular}
(C) ETD- Educação Temática Digital
Campinas, SP v.20 n.4
p.1117-1160
out./dez. 2018 


\begin{tabular}{|c|c|c|c|c|}
\hline Coelho e Costa(2016) & $\mathrm{C}$ & Faz Games & Livre & Criação de jogos educativos \\
\hline Silva (2014) & $\mathrm{C}$ & Social Games (Facebook) & Livre & Jogos para ensino de Lingua Portuguesa \\
\hline Vidani (2012) & C & EMSAVE & Livre & $\begin{array}{c}\text { Ensinar procedimentos de saúde para } \\
\text { enfermeiros em situações reais para } \\
\text { tomada de decisão }\end{array}$ \\
\hline $\begin{array}{l}\text { Bellotti, Berta e } \\
\text { Gloria (2010) }\end{array}$ & C & StoryTec & Livre & Criação de ambiente de DS nos jogos \\
\hline Park (2012) & C & $\begin{array}{l}\text { Zoombinis Mountain } \\
\text { Rescue } 2 \\
\text { Mathematics classroom }\end{array}$ & Prop & Ensino de matemática e lógica \\
\hline $\begin{array}{l}\text { Dominguez et al. } \\
\text { (2015) }\end{array}$ & C & Sim Investigator & Livre & $\begin{array}{l}\text { Aprendizagem de disciplinas do ensino } \\
\text { fundamental }\end{array}$ \\
\hline Guerreiro (2015) & $\mathrm{C}$ & Mr. Ratômico & Livre & Ensino de Química \\
\hline Sousa (2015) & C & Plinks & Livre & $\begin{array}{l}\text { Um mundo onde vivem os plinks cheio de } \\
\text { jogos }\end{array}$ \\
\hline Bordini (2016) & $\mathrm{C}$ & Musikinésia & Livre & Ensino de Música \\
\hline
\end{tabular}

Legenda: $\mathrm{C}=$ Computador, $\mathrm{M}=$ Mobile, Prop = Proprietário.

É evidente que a maioria dos pesquisadores optaram pelo uso do computador para aplicação do estudo. Entretanto, vale ressaltar que apenas a partir de 2013, provavelmente pela popularização dos smarthphones, é que se faz notar o início da utilização de dispositivos móveis. Outro destaque evidente é a opção por utilizar softwares livres. No total $86 \%$ dos pesquisadores preferiram jogos digitais gratuitos e os que optaram pelo software pago, ou seja, pelo proprietário, tentaram entender outros aspectos que não necessitavam de aplicação em grandes turmas. Essas informações são analisadas com mais profundidade no decorrer desse artigo.

\section{DISCUSSÃO DOS RESULTADOS}

O objetivo desta Revisão Sistemática da Literatura é entender como pesquisadores têm relacionado a utilização da Narrativa Digital (ND) em situações de Aprendizagem Baseada em Jogos Digitais (ABJD). Apesar da ABJD ter um grande acúmulo de pesquisas acadêmicas nos últimos 20 anos (ALVES, 2008, p.227), são poucos os artigos que tratam dos aspectos relativos à tensão das narrativas (Digital Storytelling) nos ambientes virtuais dos jogos educacionais digitais.

A tese de doutorado de Madej (2007) é um estudo que examina a percepção de crianças em relação à ND. Apesar do estudo considerar tecnologias já ultrapassadas, teoricamente a autora faz uma discussão pertinente na discussão do que é narrativa tradicional e sua diferença com a narrativa digital dos jogos de videogame. Para a autora existe um debate entre narratologistas e ludologistas no sentido de defender que os jogos digitais tenham uma narrativa linear. Entretanto, a autora afirma que "a trama linear clássica, com a sua sequência causal de eventos e tempo, não é bem suportada nos jogos digitais, porque a interatividade interfere com a sequência causal, com a linearidade, e com temporalidade" (MADEJ, 2007, p.147, tradução nossa).

\section{(C) ETD- Educação Temática Digital Campinas, SP $\quad$ v.20 n.4 $\quad$ p.1117-1160 out./dez. 2018}


Hsiao (2007) realizou uma experiência utilizando o jogo The Sim's 2. A intenção era entender se o jogador constrói uma experiência própria de jogabilidade e narrativa. Corroborando com Madej (2007), ele identificou que o jogo tem uma Interface Narrativa Interativa (INI) que "inspira os jogadores a construir e descobrir dimensões de suas identidades" (HSIAO, 2007, p.5, tradução nossa). Esse estudo vai de encontro com a ideia que a Narrativa Digital tem aspectos cognitivos diferentes da narrativa linear tradicional. Para o autor esse processo pode criar uma "autorreflexão e criar novos entendimentos e significados através dos quais se pode examinar o significado que está por trás do jogo narrativo".

Os aspectos que envolvem como o aprendiz absorve o conteúdo da ND no ambiente de ABJD é um tema que ainda envolve muita discussão acadêmica. Brom, Šisler e Slavík (2009), tratam em seu artigo de como essa transferência ocorre. Essa discussão também está em Harrold (2015) que utilizou o construtor de jogos Game Lab 3D sob a ótica do enactivismo para tentar compreender como os alunos interagem com o ambiente narrativo. Para Harrold $(2015$, p.7) "o aprendizado ocorre através de atos do aluno e a interação com objetos e eventos". Rodrigues e Bidarra (2014, p.43, tradução nossa) acreditam que a aprendizagem deve ocorrer num ambiente transmídia para que o conteúdo da ND seja transferido de forma eficaz. Isso ocorrerá "através de narrativas dinâmicas imersivas e interconectadas onde a transmídia envolve múltiplas alfabetizações, incluindo alfabetização textual, visual e midiática, bem como inteligências múltiplas".

A perspectiva de entender o processo de transmissão do conhecimento através da Narrativa Digital é alvo de praticamente todos os documentos avaliados nesta RSL. Autores ainda divergem pontualmente sobre o papel da ND aplicado ao aprendizado quando se pensa em jogos digitais educativos. E existe uma pequena inclinação para estudos baseados na Teoria Construtivista (Domínguez et al. (2015), Park (2012), Kronenberg (2013), Rubino et al. (2015), Renner (2014), Ferguson (2014), Yang e Chang (2013)). Esses estudos focam na utilização de ABJD para a construção de pensamento crítico e resolução de problemas complexos e entendem que a ND é parte fundamental para o aprendizado baseado em jogos digitais. Yang e Chang (2013, on-line) são enfáticos ao afirmarem que o "o pensamento crítico do aluno pode ser cultivado através de vários tipos de aprendizagem centrada no aluno, tais como a narrativa digital com suporte nos computadores para a resolução de problemas".

Para Hsiao (2007) as Narrativas digitais em ABJD podem propiciar a melhoria da capacidade dos alunos em criar pensamento crítico e resolução de problemas complexos. Essas narrativas agem cognitivamente de uma forma ainda não compreendida por grande parte dos estudos, que vez ou outra, ainda confundem a narrativa como o simples processo histórico de contar uma história com começo meio e fim. Nesse sentido a dissertação de Guerreiro (2015, p.92) desenvolveu um jogo chamado Mr. Ratômico para o ensino de (C) ETD-Educação Temática Digital Campinas, SP $\quad$ v.20 n.4 $\quad$ p.1117-1160 out./dez. 2018 
química em escolas públicas. $\mathrm{O}$ jogo possibilitou identificar problemas ao tentar inserir uma narrativa pouco flexível no ambiente da ABJD. O autor afirma que os jogadores querem ter "controle sobre a situação". Ou seja, "eles têm um senso real de propriedade sobre o que estão fazendo. Essa apropriação é mais rara na escola já que nos ambientes escolares o currículo e os estilos de aprendizagem usados são incompatíveis com essas ações". Sendo assim a ND, onde os aspectos cognitivos são multimodais e o ambiente altamente interativo deveria, em princípio, empoderar o estudante a ser o condutor da própria narrativa e do seu aprendizado. Entretanto, Sousa (2015) diz que em relação a aprendizagem baseada em jogos digitais o fato do jogo não obrigar ninguém a nada, por si só, é uma contraproposta aos métodos tradicionais de ensino que sempre exigem obrigações do aprendiz. Assim, imaginar ambientes de Narrativa Digital na aprendizagem é um processo em desenvolvimento que necessita de pesquisas futuras que indiquem possíveis caminhos aos gestores dos sistemas de ensino.

Os documentos analisados nesta RSL ainda sugerem dificuldades ao utilizar narrativas digitais em ABJD. Principalmente no sentido da criação de jogos educativos. Processo longo que, muitas vezes, não tem participação do professor. Em $86 \%$ dos casos os pesquisadores utilizaram jogos ou softwares de criação de jogos com plataforma livre e apenas $14 \%$ optaram por jogos proprietários. Entretanto, apenas três estudos indicaram um ambiente para desenvolver jogos digitais narrativos. Foi o caso de Berllotti, Berta e Glória (2010) que indicaram o Story Tec como ferramenta específica para a criação de jogos educativos com narrativa digital, Weiß e Müller (2008) que utilizaram uma plataforma conversacional chamada Scenejo que busca criar narrativas entre o jogador e chatbots e Yang e Chang (2013) que propõe aos alunos a criação de jogos no estilo Rolling Player Games utilizando o RPG Maker. Todos esses softwares podem ser utilizados por professores e alunos no sentido de uma produção autodidata de jogos educativos com ND.

\section{CONCLUSÃO}

A resposta para a questão de pesquisa é que a utilização de Narrativas Digitais em Ambiente de Aprendizado Baseado em Jogo Digitais ainda denota da falta de entendimento sobre os aspectos que diferenciam a narrativa linear tradicional e a narrativa digital. Ou seja, a narrativa do contador de histórias, aquela semelhante aos livros analógicos, onde o aprendiz tem papel passivo e sua contribuição ao aprendizado se limita a olhar e escutar. Não é bem aplicada quando se fala em aprendizado mediado por jogos educativos digitais. A narrativa é um termo que tem vários significados em mudança (HSIAO, 2007). Sendo assim é necessário ampliar os estudos no campo da ND e entender seus aspectos cognitivos que emolduram um novo tipo de estudante. Ou seja, aquele aprendiz, nativo digital, que tem múltiplas inteligências e a capacidade de processar informações de forma diferente dos imigrantes digitais.

(C) ETD- Educação Temática Digital Campinas, SP $\quad$ v.20 n.4 $\quad$ p.1117-1160 $\quad$ out./dez. 2018


Essa RSL pode delimitar o estado da arte da Narrativa Digital e indicar que é necessário ampliar o conhecimento e os estudos que tratem desse novo tipo de ambiente. Fato é, que imaginar uma narrativa que não tenha um começo bem definido, meio ou nem mesmo tenha um fim é um processo que demanda abstração. Uma narrativa digital invisível é parte substancial dos ambientes de ABJD (NEVILLE, 2010). Nesse ambiente existe, ao contrário das narrativas lineares, um ambiente narrativo que deverá ser interpretado de forma que gere prazer lúdico o que está diretamente enraizado no cinestésico dos aprendizes (MOULTHROP, 2004 apud NEVILLE, 2010). Os 28 estudos aqui avaliados, de forma quase total, apesar de divergirem em alguns aspectos sobre o que é narrativa digital e narrativa linear, no geral, concordam em afirmar que as Tecnologias da Informação e Comunicação transformaram o hábito milenar de se contar histórias. Dessa forma, os estudos futuros necessitam responder de que forma a ND em ABJD infere na reflexão do sujeito, contribuindo para que o mesmo obtenha o aprendizado de forma eficaz. Também é preciso supor que os professores sejam habilitados para entenderem o processo de criação de um jogo digital e os aspectos da ND necessários para que o aprendizado aconteça de forma eficaz.

Entendemos que a utilização da ND em ABJD ainda necessita de uma classificação própria que Ihe diferencie da narrativa linear tradicional ou de comparações com narrativa de livros digitais ou enredos roteirizados parecidos com os filmes. É necessário que os jogos digitais narrativos, sejam entendidos como ambientes que infiram o aprendizado de forma indireta ou mesmo imperceptível. Tarefa que necessitará de mais estudos e criação de plataformas como o StoryTec que propiciem ao professor criar seus próprios desafios de games focados na Narrativa Digital.

\section{REFERÊNCIAS}

ALVES, Lynn Rosalina Gama; CARDOSO, Hugo Saba Pereira; SOUZA, Claudio Reynaldo Barbosa de (Org.). Interfaces entre games, pesquisa \& mercado. Salvador: Edufba, 2016. 272 p.

ALVES, Lynn Rosalina Gama. Games e educação a construção de novos significados. Revista portuguesa de pedagogia, n. 42-2, p. 225-236, 2008.

AUGUSTIN, Thomas et al. The simplified updating rule in the formalization of digital educational games. Journal of Computational Science, v. 4, n. 4, p.293-303, jul. 2013. http://dx.doi.org/10.1016/i.jocs.2012.08.020

BELL, K.R. Online 3.0 - The rise of the gamer educator the potential role of gamification in online education. Ann Arbor: Univ. of Pennsylvania, 2014. (Order No. 3635727).

BELLOTTI, Francesco; BERTA, Riccardo; GLORIA, Alessandro de. Designing effective serious games: opportunities and challenges for research. International Journal of Emerging

(C) ETD-Educação Temática Digital Campinas, SP $\quad$ v.20 n.4 $\quad$ p.1117-1160 $\quad$ out./dez. 2018


Technologies in Learning (IJET), [s.I.], v. 5, n. 3, p.22-35, nov. 2010. International Association of Online Engineering (IAOE). http://dx.doi.org/10.3991/ijet.v5s3.1500

BORDINI, Rogério Augusto. Formação de professores e tecnologia digital: um estudo sobre a utilização do jogo musikinésia na educação musical. 2016. 112 f. Dissertação (Mestrado) Curso de Educação, Universidade Federal de São Carlos, São Carlos, 2016.

BROM, Cyril; SISLER, Vít; SLAVÍK, Radovan. Implementing digital game-based learning in schools: augmented learning environment of 'Europe 2045'. Multimedia Systems, [s.I.], v. 16, n. 1, p.23-41, nov. 2009. Springer Nature. http://dx.doi.org/10.1007/s00530-009-0174-0 Disponível em: http://link-springer-

com.ez46.periodicos.capes.gov.br/article/10.1007/s00530-009-0174-0 . Acesso em: 28 out. 2016.

COELHO, Patricia Margarida Farias; COSTA, Marcos Rogério Martins. Uma ferramenta digital que faz games educativos: o contexto brasileiro de ensino e aprendizagem. Ried, [s.l.], v. 19, n. 2, p.53-70, mar. 2016. UNED - Universidad Nacional de Educacion a Distancia. http://dx.doi.org/10.5944/ried.19.2.14758

DOMÍNGUEZ, Arturo Hernández et al. Um jogo digital baseado no construcionismo. Revista Brasileira de Informática na Educação, [s.I.], v. 23, n. 2, p.175-189, nov. 2015. Comissão Especial de Informática na Educação. http://dx.doi.org/10.5753/rbie.2015.23.02.175

FERGUSON, T.L.K. Mathematics achievement with digital game-based learning in high school Algebra 1 classes. 2014. 91. (Order No. 3613196) - Ann Arbor: Liberty Univ., 2014.

FREIRE, Patrícia de Sá. Aumente a qualidade e quantidade de suas publicações científicas: manual para elaboração de projetos e artigos científicos. Curitiba: Crv, 2013.

FREIRE, Paulo. Pedagogia do oprimido. 11. ed. Rio de Janeiro: Paz e Terra, 1987. 107 p. (O mundo, hoje.).

FOOKS, L.R. Gaming to learn: Learning styles and game strategies for online mathematics success. 2014. 142. (Order No. 3613847) - Capella University, Ann Arbor, 2014.

GALVÃO, Taís Freire; PEREIRA, Mauricio Gomes. Revisões sistemáticas da literatura: passos para sua elaboração. Epidemiologia e Serviços de Saúde, [s.I.], v. 23, n. 1, p.183-184, mar. 2014. Instituto Evandro Chagas. http://dx.doi.org/10.5123/s1679-49742014000100018 Disponível em: http://scielo.iec.pa.gov.br/pdf/ess/v23n1/v23n1a18.pdf . Acesso em: 17 nov. 2016.

GÖBEL, Stefan; MEHM, Florian. Personalized, Adaptive Digital Educational Games using Narrative Game-Based Learning Objects. In: BREDL, Klaus; BÖSCHE, Wolfgang. Serious games and virtual worlds in education, professional development, and healthcare. Karlsruhe: Igi Global, 2013. p. 74-84.

(C) ETD-Educação Temática Digital Campinas, SP $\quad$ v.20 n.4 $\quad$ p.1117-1160 out./dez. 2018 
GOMES, Isabelle Sena; CAMINHA, Iraquitan de Oliveira. Guia para estudos de revisão sistemática: uma opção metodológica para as Ciências do Movimento Humano. Movimento (ESEF/UFRGS), v. 20, n. 1, p. 395-411, 2014.

GUERREIRO, Manoel Augusto da Silva. Os efeitos do Game Design no processo de criação de jogos digitais utilizados no ensino de Química e Ciências: o que devemos considerar?. 2015. 297 f. Dissertação (mestrado) - Universidade Estadual Paulista Julio de Mesquita Filho, Faculdade de Ciências, 2015. Disponível em: http://hdl.handle.net/11449/126484

HARROLD, D.J. Game on: a qualitative case study on the effects of gamified curriculum design on student motivational learning habits. 2015. 192. (Order No. 3691842) - Ann Arbor: Robert Morris Univ., 2015.

HSIAO, H. "The Sims 2": reflective learning and identity construction. 2007. 218. (Order No. 3284941) - Ann Arbor: The Pennsylvania State University, 2007.

JENKINS, A.S. An investigative high-level design of an electric bass tutoring system integrating game elements. 2011. 133. (Order No. MR74804). Ann Arbor: McGill Univ. (Canada), 2011.

KRONENBERG, Felix A. Technology and the changing nature of narratives in language learning and teaching. In: Increasing student engagement and retention using multimedia technologies: video annotation, multimedia applications, videoconferencing and transmedia storytelling, [s.l.], p.95-119, jan. 2013. Emerald. http://dx.doi.org/10.1108/s2044-9968(2013)000006f007

LEE, M.J. Teaching and engaging with debugging puzzles. 2015. 261. (Order No. 3725063) Ann Arbor: Univ. of Washington, 2015.

LÉVY, Pierre. As tecnologias da inteligência: o futuro do pensamento na era da informática. 2. ed. São Paulo: Ed. 34, 2010. 128.

LIMA, Carmen Lúcia Castro. Economia criativa e a sociedade do conhecimento: notas sobre o segmento de jogos digitais. In: ALVES, Lynn Rosalina Gama; CARDOSO, Hugo Saba Pereira; SOUZA, Claudio Reynaldo Barbosa de (Org.). Interfaces entre games, pesquisa $\&$ mercado. Salvador: Edufba, 2016. p. 219-238.

LITTLE, T.W. Effects of digital game-based learning on student engagement and academic achievement. 2015. 146. (3721273). Ann Arbor: Lamar Univ., 2015.

MADEJ, K.S. Characteristics of early narrative experience: connecting print and digital game. 2007. 200. (Order No. NR41016) - Ann Arbor: Simon Fraser Univ. (Canada), 2007.

NETTO, Marinilse. Aprendizagem na EaD, mundo digital e 'gamification'. In: FADEL, Luciane Maria et al (Org.). Gamificação na educação. São Paulo: Pimenta Cultural, 2014. p. 98-121. 
NEVILLE, David O. Structuring narrative in 3d digital game-based learning environments to support second language acquisition. Foreign Language Annals, [s.l.], v. 43, n. 3, p.446-469, ago. 2010. Wiley-Blackwell. http://dx.doi.org/10.1111/j.1944-9720.2010.01092.x.

NEWZOO (São Francisco). The brazilian gamer / 2017: CONSUMER INSIGHTS. 2017. Disponível em: https://newzoo.com/insights/infographics/the-brazilian-gamer-2017/ Acesso em: 27 mar. 2018.

PADILLA-ZEA, Natalia et al. Modeling storytelling to be used in educational video games. Computers In Human Behavior, [s.I.], v. 31, p.461-474, fev. 2014. Elsevier BV. http://dx.doi.org/10.1016/i.chb.2013.04.020

PARK, Hyungsung. Relationship between motivation and student's activity on educational game. International Journal of Grid and Distributed Computing, [s.i], v. 5, n. 1, p.101-114, mar. 2012. Mensal. Disponível em: http://www.sersc.org/journals/IJGDC/vol5 no1/8.pdf . Acesso em: 10 nov. 2016.

PEREIRA, Mauricio Gomes; GALVÃO, Taís Freire. Extração, avaliação da qualidade e síntese dos dados para revisão sistemática. Epidemiologia e Serviços de Saúde, [s.I.], v. 23, n. 3, p.577-578, set. 2014. Instituto Evandro Chagas. http://dx.doi.org/10.5123/s167949742014000300021.

PRENSKY, Marc. Aprendizagem baseada em jogos digitais. São Paulo: Senac, 2012.

RAMPAZZO, Lino. Metodologia científica: para alunos dos cursos de graduação e pósgraduação. 7. ed. São Paulo: Loyola, 2013.

RENNER, J.C. Does augmented reality affect high school students' learning outcomes in chemistry?. 2014. 197. (Order No. 3628589) - Ann Arbor: Grand Canyon Univ., 2014.

RODRIGUES, Patrícia; BIDARRA, José. Transmedia Storytelling and the Creation of a Converging Space of Educational Practices. International Journal of Emerging Technologies In Learning (IJET), [s.I.], v. 9, n. 6, p.42-48, dez.2014. International Association of Online Engineering (IAOE). http://dx.doi.org/10.3991/ijet.v9i6.4134

RUBINO, Irene et al. Integrating a Location-Based Mobile Game in the Museum Visit. Journal on Computing and Cultural Heritage, [s.I.], v. 8, n. 3, p.1-18, maio.2015. Association for Computing Machinery (ACM). http://dx.doi.org/10.1145/2724723

SILVA, Emanuel Feliciano da. Multiletramentos: os games como interface para o ensino de Língua Portuguesa. 2014. 137 f. Dissertação (Mestrado) - Curso de Mestrado Profissional em Formação de Pessoas, Universidade Federal da Paraíba, Campina Grande, 2014.

SOUSA, Carla Alexandre Barboza de. 0 jogo em jogo: a contribuição dos games no processo de aprendizagem de estudantes do Ensino Fundamental. 2015. 156 f. Dissertação (Mestrado) - Curso de Educação Matemática e Tecnológica, Universidade Federal de Pernambuco, Recife, 2015.

(C) ETD-Educação Temática Digital Campinas, SP v.20 n.4 p.1117-1160 out./dez. 2018 
SWAN, R.H. Deriving operational principles for the design of engaging learning experiences. 2008. 217. (Order No. 3319394) - Ann Arbor: Brigham Young Univ., Ann Arbor, 2008.

TOMASO, P. A quantitative assessment of the effect of games on learning. 2014. 92. (Order No. 3628697) - Ann Arbor: Colorado Technical Univ., 2014.

VIDANI, Alberto Cabas. An architecture supporting the development of serious games for scenario-based training and its application to advanced life support. 2012. $98 \mathrm{f}$. Tese (Doutorado) - Curso de Informática, Università Degli Studi di Udine, Udine, 2012. Disponível em: https://dspace-uniud.cilea.it/handle/10990/124 . Acesso em: 01 nov. 2016.

WEIß, Sebastian A.; MÜLLER, Wolfgang. Learning with Interactive Stories. IFIP - The International Federation For Information Processing, [s.I.], p.321-328, 2008. Springer Science + Business Media. http://dx.doi.org/10.1007/978-0-387-09729-9 47.

YANG, Ya-ting Carolyn; CHANG, Chao-hsiang. Empowering students through digital game authorship: Enhancing concentration, critical thinking, and academic achievement.

Computers \& Education, [s.I.], v. 68, p.334-344, out. 2013. Elsevier BV.

http://dx.doi.org/10.1016/i.compedu.2013.05.023.

\section{Agradecimentos}

Essa RSL foi possível devido ao apoio da Fundação de Amparo à Pesquisa do Estado de Santa Catarina (FAPESC) e graças à equipe do Laboratório de Mídia e Conhecimento (Labmídia) da Universidade Federal de Santa Catarina (Campus Araranguá-SC). 